\title{
Prevalence of periodontopathogens and Candida spp. in smokers after nonsurgical periodontal therapy - a pilot study
}

\section{Gabriela Alessandra da Cruz Galhardo CAMARGO(a) \\ Mariana Gouvêa Latini ABREU(a) Renata dos Santos CORDEIRO(a) Letícia de Farias WENDEROSCKY(a) Cristiane DUQUE(b)}

(a) Universidade Federal Fluminense - UFF, Department of Periodontology, Nova Friburgo, RJ, Brazil.

(b) Universidade Estadual Paulista - UNESP, Araçatuba Dental School, Department of Pediatric Dentistry and Public Health, Araçatuba, SP, Brazil.

Declaration of Interests: The authors certify that they have no commercial or associative interest that represents a conflict of interest in connection with the manuscript.

Corresponding Author:

Cristiane Duque

E-mail: cduque@foa.unesp.br

DOI: 10.1590/1807-3107BOR-2016.vol30.0092

Submitted: Dec 18, 2015

Accepted for publication: Apr 26, 2016

Last revision: May 30, 2016

\begin{abstract}
This pilot study aimed to evaluate the influence of smoking on clinical and microbiological parameters after nonsurgical periodontal therapy. Forty-eight subjects were grouped into smokers $(\mathrm{SM}, \mathrm{n}=24)$ and nonsmokers (NS, $\mathrm{n}=24$ ) and paired according to gender, age, ethnicity, and periodontal status. Both groups received oral hygiene education and scaling and root planing. Clinical evaluation was performed using plaque index (PI), bleeding on probing (BOP), pocket probing depth (PPD), gingival recession (GR), and clinical attachment level (CAL) before instrumentation (baseline) and at 3 and 6 months. The prevalence of Aggregatibacter actinomycetemcomitans, Porphyromonas gingivalis, Tannerella forsythia, Candida albicans, Candida glabrata, Candida tropicalis, and Candida dubliniensis in subgingival biofilm was determined by polymerase chain reaction. The data were statistically analyzed considering $\mathrm{p}<0.05$. Clinical conditions improved between baseline and 3 months after periodontal treatment. However, NS had a better clinical response, presenting greater PPD reduction and CAL increase in comparison to SM. Periodontal treatment reduced the levels of $P$. gingivalis, A. actinomycetemcomitans, and T. forsythia individually after 3 months for the NS group and after 6 months for both groups. The prevalence of Candida species was markedly higher in SM than in NS at all time points evaluated. Periodontopathogens associated or not with C. albicans or C. dubliniensis were more prevalent in SM than in NS at baseline and after 3 months. It was concluded that smoking impairs clinical and microbiological responses to periodontal therapy. Periodontopathogens combined or not with some Candida species are resistant to short-term periodontal therapy in SM.
\end{abstract}

Keywords: Periodontal Diseases; Smoking; Bacteria, Anaerobic; Candida; Periodontal Debridement.

\section{Introduction}

Several countries have been investing in numerous initiatives and in government policies to control tobacco consumption; however, cigarette smoking still remains prevalent in the world, with more than 1 billion smokers, and more than 8 million deaths from one or more diseases related to tobacco use will have occurred every year by $2030 .{ }^{1}$ In addition to systemic damages, such as increased risk for the development of chronic obstructive pulmonary disease, cardiovascular complications, 
and diverse forms of malignant tumors, especially lung cancer, tobacco smoking has been considered the most important risk factor for periodontitis among all lifestyle factors. ${ }^{1,2}$

Several studies all around the world have pointed out a strong association between tobacco use/smoking habit and periodontal disease, evidenced by greater clinical attachment loss, gingival recession, and tooth loss, ${ }^{3,4,5}$ as well as by reduced bone height and density. ${ }^{6,78}$ The harmful effects of smoking on periodontal tissues are caused by cytotoxic and vasoactive substances present in tobacco, including nicotine, carbon monoxide, and reactive oxygen species. These substances cause oxidative stress and alter immunoinflammatory responses, reducing the functional activity of leukocytes, macrophages, lymphocytes, and other immune cells, and impairing wound healing and microbial identification. ${ }^{9}$

There is no consensus about the relationship between smoking habit and increased risk of subgingival infection with periodontal pathogens. Some authors have reported a high number of pathogens belonging to the red and orange complexes at periodontal sites in smokers ${ }^{10,11,12}$ while others have not found any differences between smokers and nonsmokers. ${ }^{13,14,15}$ The presence of cigarette components could alter the oral environment and trigger the colonization of periodontal sites by uncommon pathogens, as shown by Kamma et al., ${ }^{16}$ who compared the microbial profile of smokers and nonsmokers in a group of patients with early-onset periodontitis and found significant levels of E. coli, C. albicans, S. aureus, and other exogenous pathogens in smokers' microbiota.

Although the most usual periodontal treatment consists of nonsurgical therapy with scaling and root planing, which is widely known for improving clinical and microbiological parameters, previous studies have demonstrated worse clinical response in smokers when compared to nonsmokers, suggesting that tobacco use could impair the response to therapeutic intervention..$^{17,18,19}$ Few studies have evaluated longitudinal clinical and microbiological status of smokers undergoing periodontal maintenance therapy, yielding controversial results, with no difference in disease progression ${ }^{20}$ or higher prevalence of tooth loss among smokers ${ }^{21,22}$ than among nonsmokers. This pilot study aimed to evaluate the influence of smoking on clinical and microbiological parameters after nonsurgical periodontal therapy. The null hypothesis was that short-term periodontal therapy would not improve clinical and microbiological parameters in smokers in comparison with nonsmokers.

\section{Methodology}

\section{Study population}

Forty-eight patients (24 smokers - SM and 24 nonsmokers - NS), aged 27 to 70 years, were selected to participate in this study. All subjects were recruited from the Department of Periodontology, School of Dentistry, Universidade Federal Fluminense - UFF, Nova Friburgo, Rio de Janeiro State, Brazil, for a period of over 6 months between 2010 and 2013. The study protocol (CAAE - 0070.0.258.000-10) was approved by the Ethics Committee of the School of Medicine, Universidade Federal Fluminense - UFF, and registered at the US National Institutes of Health Clinical Trials (Register number: NCT02581319 at http://www. clinicaltrials.gov). Prior to participation, the study purpose and procedures were fully explained to all patients, who eventually signed a written informed consent form pursuant to the Declaration of Helsinki. The initial sample size of 25 patients per group was chosen based on standard deviations from a previous study, ${ }^{23}$ an effect size (gingival index [GI], a minimum detectable change of $10 \%$ ), a power of $80 \%$, a significance level of $5 \%$, and a loss to follow-up rate of up to $20 \%$.

Medical and dental histories were taken and patients were clinically evaluated during prescreening visits. The inclusion criteria were: presence of periodontal disease in at least two teeth in different arches, bleeding on probing on these sites where probing depth was $\geq 5 \mathrm{~mm}$; and radiographic evidence of bone loss ranging from 30 to $50 \% .{ }^{24}$ Subjects were considered smokers if they had been smoking 10 or more cigarettes a day for at least 2 years. Smoking habit was confirmed in each visit and individuals who had stopped smoking were excluded. Former smokers were not included in the control group.

The exclusion criteria were: patients with systemic diseases; diabetes; osteoporosis; pregnant lactating 
females; use of immunosuppressants, phenytoin, cyclosporine, calcium channel blockers, or any use of antibiotics or nonsteroidal anti-inflammatory drugs in the past 3 months; and any medical conditions requiring immunotherapy or patients diagnosed as HIV+ or with AIDS, which could interfere with periodontal status. ${ }^{24}$

\section{Clinical examination and periodontal therapy ${ }^{24}$}

Prior to clinical examination, a questionnaire was applied to obtain information about patients' smoking habits (pack years and daily cigarette consumption) at baseline and at 3 and 6 months. An experienced periodontist (GACGC) assessed the clinical periodontal parameters, including plaque index (PI), bleeding on probing (BOP), pocket probing depth (PPD), gingival recession (GR), and clinical attachment level (CAL) using a PCP15 periodontal probe (PCP-UNC15, Hu-Friedy, Chicago, USA), at six sites per tooth and on all teeth, except third molars. ${ }^{24}$ The intra-rater reliability for the categorical variables (PI, BOP) at tooth level was calculated by the kappa statistic, which was equal to 0.75 . The reproducibility of continuous variables (PD, GR, and CAL) was 0.70 as indicated by the intraclass correlation coefficient (ICC).

At baseline, quadrant scaling and root planing (Q-SRP) was performed weekly under local anesthesia using periodontal curettes (American Eagle, GraceyAcess Curettes, Missoula, USA) and ultrasonic scalers (Cavitron, Dentisply, New York, USA). The maintenance therapy included professional plaque control and monthly Q-SRP in recurrent periodontal pockets throughout the study period (6 months). No teeth needed to be extracted during therapy. A different clinician (MGLA) conducted the periodontal treatment. The examiner (GACGC) had no access to previous recordings. All subjects received oral hygiene instructions for home care procedures (toothbrushing technique, interdental cleaning, and use of tongue scrapers).

\section{Microbiological sampling}

After clinical measurements, supragingival biofilm was removed with sterile gauze. Subgingival samples were collected from each subject at the sites with PPD $\geq 5 \mathrm{~mm}$ using a sterile periodontal curette. Pooled biofilms from each site were placed in microtubes containing Tris-EDTA buffer (10 mM Tris-Hcl, $0.1 \mathrm{mM}$ EDTA, $\mathrm{pH} 7.5$ ) and stored at $-20^{\circ} \mathrm{C}$ to be microbiologically analyzed by polymerase chain reaction (PCR).

\section{Microbiological evaluation - polymerase chain reaction (PCR)}

The DNA was extracted using a protocol originally described by Sardi et al. ${ }^{24}$ and quantified in a spectrophotometer at $260 \mathrm{~nm}$ (Genesys 10UV, Rochester, USA) in order to obtain a standard concentration of $100 \mathrm{ng} / \mathrm{mL}$ and stored at $-20^{\circ} \mathrm{C}$ for subsequent PCR reactions. Briefly, the samples were submitted to a lysis solution (extraction buffer and proteinase $\mathrm{K}$ ) and then purified using chloroform:isoamyl-alcohol followed by DNA precipitation with isopropanol and $70 \%$ ethanol. The DNA was resuspended in TE buffer $(10 \mathrm{mM}$ Tris- $\mathrm{HCl}$, $0.1 \mathrm{mM}$ EDTA, pH 7.5, with $10 \mu \mathrm{g} / \mathrm{mL}$ RNAse). Molecular microbial identification was carried out by PCR with specific primers for Aggregatibacter actinomycetemcomitans, ${ }^{25}$ Porphyromonas gingivalis, ${ }^{26}$ Tannerella forsythia,${ }^{27}$ Candida albicans, ${ }^{24}$ Candida glabrata, ${ }^{24}$ Candida tropicalis, ${ }^{24}$ and Candida dubliniensis. ${ }^{28}$ PCR amplification was performed with a thermocycler AmpliTherm TX96 Gradient (Axygen, NY, USA) under thermal conditions specific for each pair of primers. The PCR products were separated by $2 \%$ agarose gel electrophoresis and Tris-borate-EDTA running buffer ( $\mathrm{pH}$ 8.0). The molecular mass ladder (100 bp DNA ladder, Gibco, Grand Island, USA) was included for running in agarose gel electrophoresis. The DNA was stained with $0.1 \mu \mathrm{l}$ of SYBR Safe/mL (Invitrogen, USA) and visualized under UV light (Pharmacia LKB-MacroVue, San Gabriel, USA). Photographs were taken (Image Mater - LISCAP, VDS, Pharmacia Biotech Piscataway, USA) and analyzed.

\section{Statistical analysis}

The statistical analysis was performed using SPSS Statistics, version 17.1 (IBM Inc., Chicago, USA). Patient characteristics (age, gender, and race) and clinical parameters (PI, BOP, PPD, GR, and $\mathrm{CAL})$ were compared between smokers (SM) and nonsmokers (NS) at baseline using Student's t test (age) and chi-square test (gender/race) and the Mann 
Whitney test, respectively. The percentages of sites with A. actinomycetemcomitans, P. gingivalis, T. forsythia, C. albicans, C. glabrata, C. tropicalis, and C. dubliniensis were compared between the SM and NS groups by applying the chi-square test. In relation to periodontal treatment, subjects from both groups (SM and NS) were compared at baseline and at 3 and 6 months using Wilcoxon's test for clinical evaluation and the Mann-Whitney test for microbiological analysis. The differences were statistically significant when $\mathrm{p}<0.05$.

\section{Results}

\section{Clinical results}

There was no statistical difference betweenSMand NS whenage (means/SDSM $=44.66 \pm 10.22 ; \mathrm{NS}=53.08 \pm 10.26$, $\mathrm{p}=0.846)$, gender $(\mathrm{SM}=50 \%$ female $/ \mathrm{male} ; \mathrm{NS}=54.3 \%$ female and $45.7 \%$ male), and race (SM $=100 \%$ and NS $=83.3 \%$ white people) were considered. Means \pm SD of cigarette consumption were $19.83 \pm 10.1$ cigarettes per day and length (means/SD) of smoking habit was $24 \pm 11.73$ years. Twenty percent of SM had quit smoking and were excluded from the study. By comparing SM and NS at baseline, both groups had a similar periodontal status (Table 1). Clinical conditions improved (decrease in PI and BOP indexes and in PPD and CAL measurements) in both groups, when baseline and 3 months after periodontal treatment were compared. Significant reductions were noted for PI, PPD, and CAL for NS and for PI, BOP, PPD, and CAL for SM, when the results for baseline and 6 months were compared.

Table 1. Clinical evaluation of smokers in comparison with non-smokers at baseline and after 3 and 6 months of periodontal therapy. Values express in medians (percentile 25-percentile 75).

\begin{tabular}{|c|c|c|c|}
\hline \multirow{2}{*}{ Variables } & \multicolumn{2}{|c|}{ Groups } & \multirow{2}{*}{ p-value* } \\
\hline & Non-smokers (NS) & Smokers (SM) & \\
\hline \multicolumn{4}{|l|}{ PI (\%) } \\
\hline Baseline & $41.22(11.5-50)^{a}$ & $57.42(36.37-85.3)^{a}$ & 0.051 \\
\hline 3 months & $11.4(4.76-26.5)^{b}$ & $37.5(19.84-66.7)^{b}$ & 0.001 \\
\hline 6 months & $11.21(4.46-42.4)^{a}$ & $32.89(11.04-66.16)^{\mathrm{b}, \mathrm{c}}$ & 0.075 \\
\hline \multicolumn{4}{|l|}{$\mathrm{BOP}(\%)$} \\
\hline Baseline & $23.07(11.5-46.39)^{a}$ & $22.64(12.95-62.05)^{a}$ & 0.726 \\
\hline 3 months & $8.77(3.4-25.69)^{\mathrm{b}}$ & $24.6(13.49-31.33)^{\mathrm{b}}$ & 0.025 \\
\hline 6 months & $18.92(15.84-23.58)^{a}$ & $13.97(9.35-29.48)^{c}$ & 0.393 \\
\hline \multicolumn{4}{|l|}{$\mathrm{PD}(\mathrm{mm})$} \\
\hline Baseline & $5.04(5-5.24)^{a}$ & $5(5-5.23)^{a}$ & 0.722 \\
\hline 3 months & $3(2.7-3.84)^{b}$ & $3.85(3.33-4.3)^{\mathrm{b}}$ & 0.04 \\
\hline 6 months & $3(2.42-3.25)^{b, c}$ & $3.41(2.73-4)^{\mathrm{b}, \mathrm{c}}$ & 0.353 \\
\hline \multicolumn{4}{|l|}{$\mathrm{GR}(\mathrm{mm})$} \\
\hline Baseline & $1.75(0-2.49)^{a}$ & $1.89(0-2.51)^{a}$ & 0.795 \\
\hline 3 months & $1.75(0-2.67)^{a}$ & $1.9(0-2.5)^{a}$ & 0.977 \\
\hline 6 months & $0.5(0-3)^{a}$ & $1.35(0-2.12)^{a}$ & 0.971 \\
\hline \multicolumn{4}{|l|}{$\mathrm{CAL}(\mathrm{mm})$} \\
\hline Baseline & $6.75(5-7.76)^{a}$ & $6.94(5.25-7.56)^{a}$ & 0.925 \\
\hline 3 months & $5.5(2.87-6.51)^{\mathrm{b}}$ & $5.4(4-6.5)^{\mathrm{b}}$ & 0.370 \\
\hline 6 months & $3.5(2.67-6)^{b, c}$ & $4.5(3.81-5.42)^{\mathrm{b}, \mathrm{c}}$ & 0.481 \\
\hline
\end{tabular}

*The significance of differences between groups at each time point was assessed using Mann-Whitney test. p-values with a statistically significant difference are bold; 'Different lower letters show statistical difference among time points, considering each condition separately (NS and SM), according to Wilcoxon test. 
There was no statistical difference between the groups in periodontal parameters when 3 and 6 months were compared, except for BOP in SM patients. Medians of PI, BOP, and PPD were lower at 3 months in NS compared to SM. The same was observed for PI and GR at 6 months, indicating that NS had a better response to periodontal treatment than SM (Table 1). PPD reduction and CAL increase in SM and NS at the three time points of periodontal treatment are shown in Table 2. Statistical differences between the groups were observed for both criteria (PPD and CAL) when comparing baseline and 3 months of treatment.

\section{Microbiological results}

Overall, periodontal treatment reduced the frequency of periodontopathogens ( $P$. gingivalis, A. actinomycetemcomitans, and T. forsythia) only in the NS group and in both groups after 3 and 6 months, respectively. The prevalence of $C$. albicans and $C$. dubliniensis among SM did not change after periodontal treatment at any time points. At 3 months, the frequency of $C$. albicans significantly increased and the frequency of $C$. dubliniensis decreased in comparison with baseline. C. glabrata was not detected in NS patients and its prevalence decreased in SM patients during the study period. The frequency of Candida tropicalis decreased in NS but did not change in SM individuals. When comparing SM and NS, the percentages of A. actinomycetemcomitans and C. albicans were lower in NS than in SM at baseline. There was no statistical difference in the frequency of microorganisms between the groups by comparing, 3 and 6 months of treatment, except for A. actinomycetemcomitans. The prevalence of three or more Candida species was markedly higher in SM than in NS (Figure 1). The combinations of bacteria and $C$. albicans or bacteria and C. dubliniensis are shown in Figures $2 \mathrm{~A}$ and 2B, respectively. After 6 months of treatment, no combinations of bacteria ( $P$. gingivalis, A. actinomycetemcomitans, and/or T. forsythia) and C. albicans or C. dubliniensis were found. The combination of bacteria and C. albicans was evidenced at baseline and at 3 months after treatment for SM and only at baseline for NS. Statistical difference was observed between NS and SM at baseline and after 3 months of treatment (Figure 2A). The same was observed for the combinations of A. actinomycetemcomitans $+P$. gingivalis $+C$. dubliniensis and A. actinomycetemcomitans + T. forsythia $+C$. dubliniensis. Statistical difference was observed for all combinations of bacteria and $C$. dubliniensis when baseline and 6 months of treatment were compared (Figure 2B).

\section{Discussion}

The present study demonstrated that conventional periodontal treatment was clinically effective for SM and NS. However, NS had a better response to periodontal treatment, with a greater PPD reduction and CAL increase than SM after 3 and 6 months of

Table 2. Medians (percentile 25-percentile 75) of PD reduction and CAL gain from smokers and non-smokers at three time points of periodontal treatment.

\begin{tabular}{lccc}
\hline Variables & \multicolumn{2}{c}{ Groups } & P-value* \\
\cline { 2 - 3 } & Non Smokers (NS) & Smokers (SM) & \\
\hline PD (mm) & $2.09(1.67-2.5)$ & $1.34(1-2)$ & $\mathbf{0 . 0 2 4}$ \\
Baseline to 3 months & $0.48(-0.67-1.12)$ & $0.3(0-0.5)$ & 0.455 \\
3 months to 6 months & $2.41(1.82-3.83)$ & $1.95(1.52-2.3)$ & $\mathbf{0 . 0 3 5}$ \\
Baseline to 6 months & & & $\mathbf{0 . 0 4 3}$ \\
CAL (mm) & $2.01(1.64-2.56)$ & $1.51(0.79-2.25)$ & 0.387 \\
Baseline to 3 months & $0.69(-0.7-2.0)$ & $0.31(0.08-0.71)$ & $\mathbf{0 . 0 3 5}$ \\
3 months to 6 months & $3(2-3.99)$ & $2.2(1.66-2.6)$ & \\
Baseline to 6 months & & \\
\hline
\end{tabular}

*The significance of differences between groups at each time point was assessed using Mann-Whitney test. p-values with a statistically significant difference are bold. 


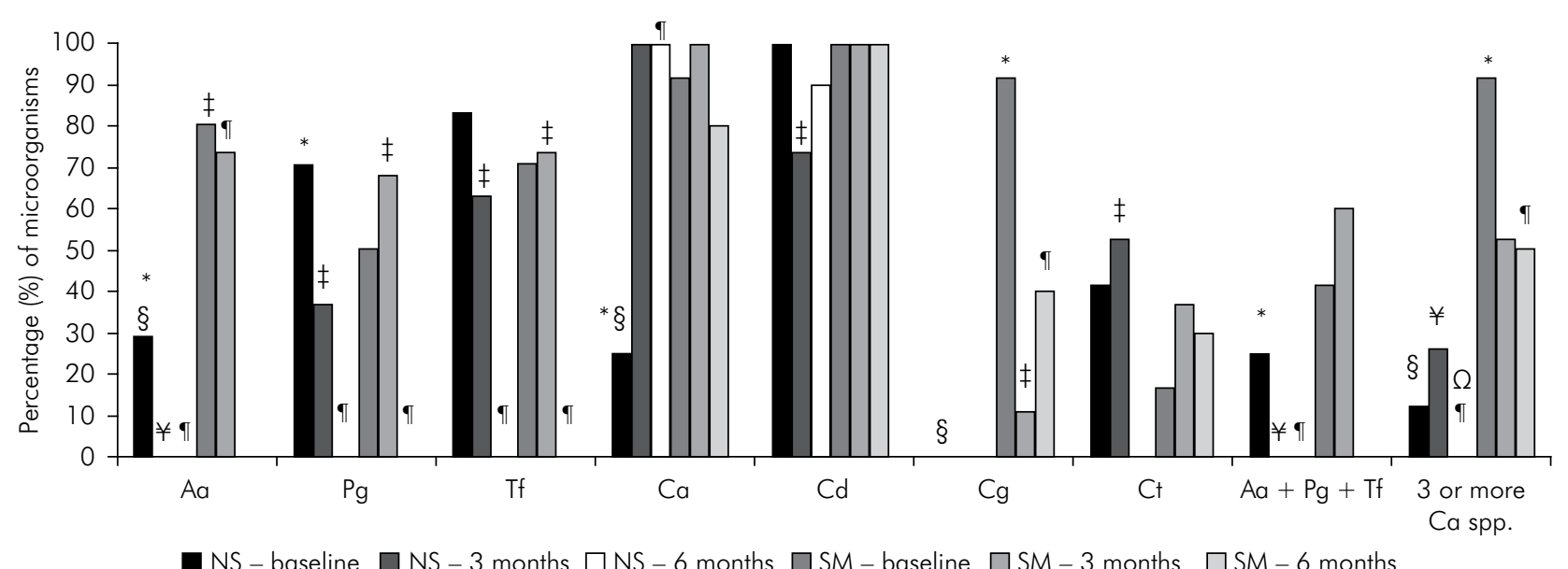

Values expressed in $\mathrm{n}(\%)$ of sites harboring the microorganism.

*Statistically significant difference between baseline and 3 months, considering each group separately (Wilcoxon's test; $p<0.05$ ); "Statistically significant difference between baseline and 6 months, considering each group separately (Wilcoxon's test; $p<0.05$ ); :Statistically significant difference between 3 months and 6 months, considering each group separately (Wilcoxon's test; $p<0.05$ ); SStatistically significant differences between SM and NS groups for baseline (Mann Whitney test; $p<0.05$ ); ${ }^{*}$ Statistically significant differences between SM and NS groups for 3 months (Mann Whitney test; $\mathrm{p}<0.05$ ); 'Statistically significant differences between SM and NS groups for 6 months (Mann Whitney test; $p<0.05$ ).

Figure 1. Microbiological evaluation of smokers and nonsmokers at baseline and after 3 and 6 months of periodontal treatment.

evaluation. These results are consistent with previous studies $^{17,18,19}$ and confirm that smoking adversely influences the clinical response of patients after periodontal treatment. Scaling and root planing is the most usual periodontal therapy, leading to improvements in clinical parameters and to the reestablishment of the microbiota. ${ }^{29}$ Although repeated treatment of recurrent/refractory pockets in the supportive phase may produce additional reductions in PPD and in BOP and increases in clinical attachment level, the response to periodontal treatment is slower in SM than in NS.

The negative effects of smoking on the immunoinflammatory and healing systems may explain the worse response of SM to periodontal treatment. It is suspected that smoking impairs innate defenses against pathogens, alters antigen presentation, and negatively modulates the adaptive immune response. ${ }^{30}$ Cigarette smoking down-regulates the surface expression of selectin in neutrophils, altering their cellular functions and, consequently, the structural integrity of the binding site. ${ }^{31}$ Smokers also exhibited significantly lower amounts of proinflammatory cytokines (IL-1a, IL-10, IL-12) and chemokines (IL-8, monocyte chemoattractant protein-1, macrophage inflammatory protein-1) than NS. ${ }^{32,33}$
Studies have demonstrated that heavy exposure to cigarette smoking suppresses osteoprotegerin production and leads to an increased ratio of RANKL to osteoprotegerin in gingival fluid, ${ }^{33}$ which explains the smaller PPD reduction and CAL increase in SM in comparison to NS.

The suppression of some essential mediators of microbial elimination associated with the increase of other mediators involved in tissue breakdown could favor the proliferation of pathogenic microorganisms at the subgingival level, enhancing susceptibility to the development of periodontitis. ${ }^{2}$ It remains unclear whether there are differences in the subgingival biofilm composition between SM and NS. Some studies have shown no differences in the prevalence of periodontal pathogens $\mathrm{s}^{13,14,15}$ while other studies have demonstrated higher levels of periodontal pathogens belonging to the red and orange complexes ${ }^{10,11,12}$ in SM compared to NS. The present study assessed the prevalence of $A$. actinomycetemcomitans, $P$. gingivalis, and T. forsythia at baseline and at 3 and 6 months of treatment and found that only A. actinomycetemcomitans was statistically higher at baseline in SM compared to NS. This finding is in line with the results of Guglielmetti et al., ${ }^{34}$ who observed an association between smoking status and the presence of $A$. 

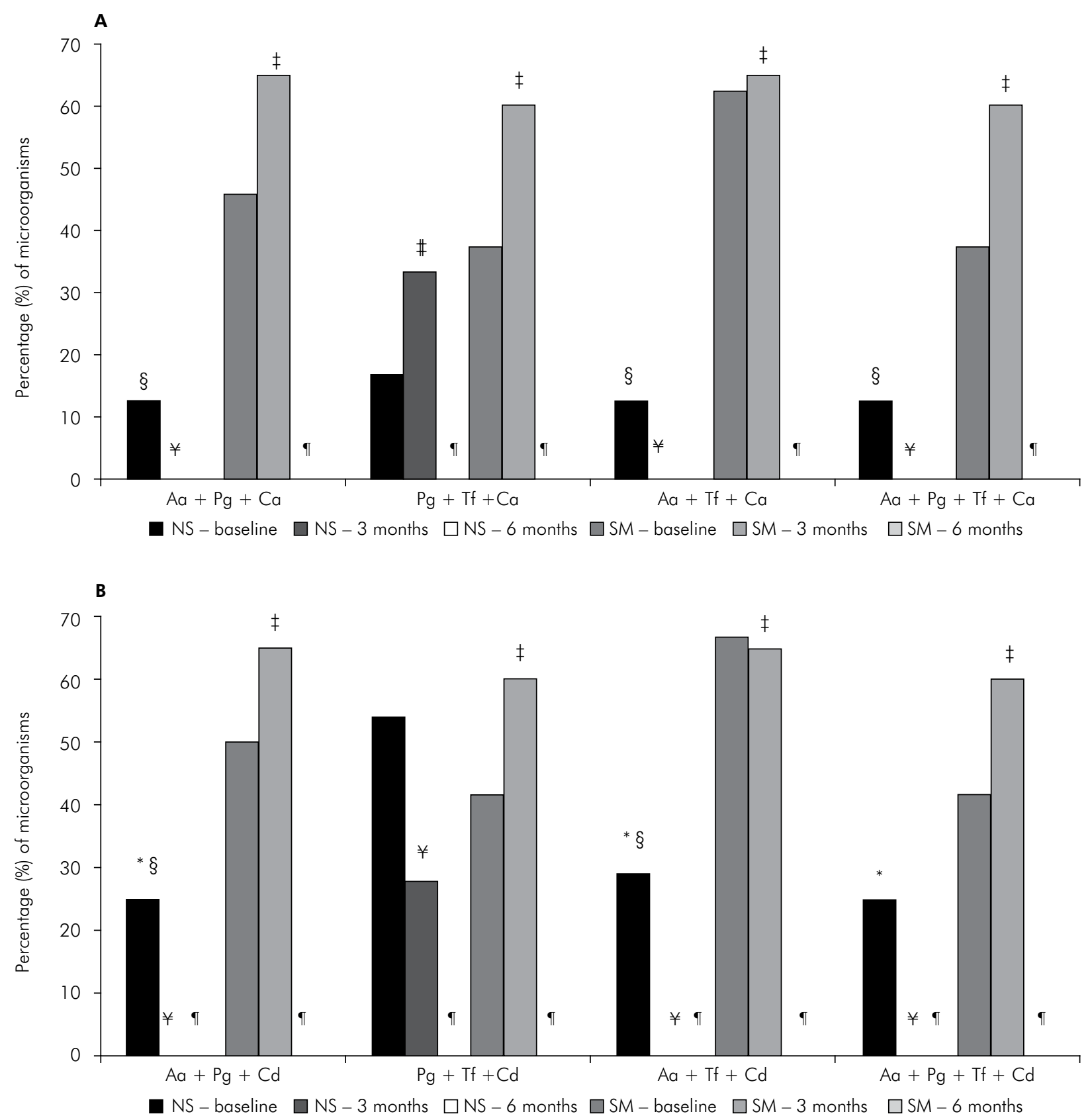

Different lower-case letters show statistical difference among time points, considering each condition separately (NS and SM), according to Kruskal-Wallis and Mann-Whitney tests; *Statistically significant difference between baseline and 3 months, considering each group separately (Wilcoxon's test; $p<0.05$ ); "Statistically significant difference between baseline and 6 months, considering each group separately (Wilcoxon's test; $p<0.05$ ); :Statistically significant difference between 3 months and 6 months, considering each group separately (Wilcoxon's test; $p<0.05$ ); §Statistically significant differences between SM and NS groups for baseline (Mann Whitney test; $p<0.05$ ); ${ }^{*}$ Statistically significant differences between SM and NS groups for 3 months (Mann Whitney test; $p<0.05)$; There were no statistical difference between SM and NS groups for 6 months (Mann Whitney test, $p<0.05$ ).

Figure 2. Combinations of bacteria and $\mathrm{Ca}(\mathrm{A})$ or $\mathrm{Cd}(\mathrm{B})$ from smokers and nonsmokers after time points of periodontal treatment. Values expressed in $\mathrm{n}(\%)$ of sites harboring the microorganism.

actinomycetemcomitans. Both groups presented favorable changes in the subgingival microbiota after treatment; however, a significant reduction in the percentage of bacterial pathogens was observed only after
6 months for SM and after 3 and 6 months for NS. Other studies have also found smaller reductions in the subgingival levels of periodontopathogens among NS after short-term conventional treatment 
(scaling and root planing). ${ }^{13,35}$ In the current study, the reduction in the levels of periodontopathogens ( $A$. actinomycetemcomitans, P. gingivalis, and T. forsythia) in both groups (SM and NS) was 100\% after 6 months of therapy. A significant reduction of T. forsythia and $A$. actinomycetemcomitans levels in both SM and NS, absence of $P$. gingivalis among NS, and very low detection of $P$. gingivalis among SM (less than $10 \%$ ) were observed after 6 months of periodontal treatment in a study conducted by Aptzidou et al. ${ }^{14}$ The same magnitude of microbiological response was observed for P. gingivalis and T. forsythia 6 months after periodontal treatment in SM and NS by Renvert et al. ${ }^{35}$ However, these authors observed that $A$. actinomycetemcomitans increased in SM, demonstrating that this pathogen is more difficult to be eradicated among tobacco users.

The habit of cigarette smoking is clearly recognized as a predisposing factor for oral yeast carriage, possibly because it induces increased epithelial keratinization and enhancement of hydrophobicity, favoring yeast proliferation and colonization of oral tissues. ${ }^{36}$ Higher rates of Candida albicans have been found in the subgingival microbiota of patients with chronic periodontitis than in healthy individuals ${ }^{37,38}$ and the prevalence of this species is higher among $\mathrm{SM}$, as pointed by Kamma et al., ${ }^{16}$ than among NS.

\section{References}

1. Haber J, Wattles J, Crowley M, Mandell R, Joshipura K, Kent RL. Evidence for cigarette smoking as a major risk factor for periodontitis. J Periodontol. 1993;64(1):16-23. doi:10.1902/jop.1993.64.1.16

2. Nociti FH Jr, Casati MZ, Duarte PM. Current perspective of the impact of smoking on the progression and treatment of periodontitis. Periodontol 2000. 2015;67(1):187-210. doi:10.1111/prd.12063

3. Do LG, Slade GD, Roberts-Thomson KF, Sanders AE. Smoking-attributable periodontal disease in the Australian adult population. J Clin Periodontol. 2008;35(5):398-404. doi:10.1111/j.1600-051X.2008.01223.x

4. Phipps KR, Chan BK, Jennings-Holt M, Geurs NC, Reddy MS, Lewis CE et al. Periodontal health of older men: the MrOS dental study. Gerodontol. 2009;26(2):122-9. doi:10.1111/j.1741-2358.2008.00231.x

5. Susin C, Haas AN, Valle PM, Oppermann RV, Albandar JM. Prevalence and risk indicators for
Interestingly, C. albicans isolated from the subgingival plaque of patients with periodontitis adhered more to epithelial cells than did those isolated from healthy patients. ${ }^{39}$ After Candida adhesion and biofilm formation, the secretion of histolytic enzymes, such as collagenases and proteases, can degrade extracellular matrix proteins. ${ }^{24}$ Moreover, C. albicans may exacerbate periodontitis by enhancing the invasion of host cells by anaerobic bacteria such as $P$. gingivalis. ${ }^{40}$ Although it is not yet possible to precisely determine the role of Candida species in the development or progression of periodontal disease, these virulence traits may explain the increased persistence of this pathogen in the subgingival microbiota in SM.

\section{Conclusion}

Smoking impairs clinical and microbiological responses to periodontal therapy. Periodontopathogens combined or not with some Candida species are resistant to short-term periodontal therapy in SM.

\section{Acknowledgments}

The authors would like to thank Fundação de Amparo a Pesquisa do Estado Rio De Janeiro -FAPERJ for the financial support (Scholarship E-26/103.243/2012). The authors declare they have no potential conflicts of interest regarding authorship and/or publication of this article. chronic periodontitis in adolescents and young adults in south Brazil. J Clin Periodontol. 2011;38(4):326-33. doi: 10.1111/j.1600-051X.2011.01699.x

6. Rosa GM, Lucas GQ, Lucas ON. Cigarette smoking and alveolar bone in young adults: a study using digitized radiographs. J Periodontol. 2008;79(2):232-44. doi:10.1902/jop.2008.060522

7. Lima FR, Cesar-Neto JB, Lima DR, Kerbauy WD, Nogueira-Filho GR. Smoking enhances bone loss in anterior teeth in a Brazilian population: a retrospective cross-sectional study. Braz Oral Res. 2008;22(4):328-33. doi:10.1590/S1806-83242008000400008

8. Hugoson A, Rolandsson M. Periodontal disease in relation to smoking and the use of Swedish snus: epidemiological studies covering 20 years (1983-2003). J Clin Periodontol. 2011;38(9):809-16. doi:10.1111/j.1600-051X.2011.01749.x 
9. Gonçalves RB, Coletta RD, Silvério KG, Benevides L, Casati $\mathrm{MZ}$, Silva JS et al. Impact of smoking on inflammation: overview of molecular mechanisms. Inflamm Res. 2011;60(5):409-24. doi:10.1007/s00011-011-0308-7

10. Haffajee AD, Socransky SS. Relationship of cigarette smoking to the subgingival microbiota. J Clin Periodontol. 2001;28(5):377-88. doi:10.1034/j.1600-051x.2001.028005377.x

11. Eggert FM, McLeod MH, Flowerdew G. Effects of smoking and treatment status on periodontal bacteria: evidence that smoking influences control of periodontal bacteria at the mucosal surface of the gingival crevice. J Periodontol. 2001;72(9): 1210-20. doi:10.1902/jop.2000.72.9.1210

12. Kubota M, Tanno-Nakanishi M, Yamada S, Okuda K, Ishihara K. Effect of smoking on subgingival microflora of patients with periodontitis in Japan. BMC Oral Health. 2011;11:1. doi:10.1186/1472-6831-11-1

13. Darby IB, Hodge PJ, Riggio MP, Kinane DF. Clinical and microbiological effect of scaling and root planing in smoker and non-smoker chronic and aggressive periodontitis patients. J Clin Periodontol. 2005;32(2):200-6. doi:10.1111/j.1600-051X.2005.00644.x

14. Apatzidou DA, Riggio MP, Kinane DF. Impact of smoking on the clinical, microbiological and immunological parameters of adult patients with periodontitis. J Clin Periodontol. 2005;32(9):973-83. doi:10.1111/j.1600-051X.2005.00788.x

15. Kibayashi M, Tanaka M, Nishida N, Kuboniwa M, Kataoka K, Nagata H et al. Longitudinal study of the association between smoking as a periodontitis risk and salivary biomarkers related to periodontitis. J Periodontol. 2007;78(5):859-67. doi:10.1902/jop.2007.060292

16. Kamma JJ, Nakou M, Baehni PC. Clinical and microbiological characteristics of smokers with early onset periodontitis. J Periodontal Res. 1999;34(1):25-33. doi:10.1111/j.1600-0765.1999.tb02218.x

17. Johnson GK, Hill M. Cigarette smoking and the periodontal patient. J Periodontol. 2004;75(2):196-209. doi:10.1902/jop.2004.75.2.196

18. Heasman L, Stacey F, Preshaw PM, McCracken GI, Hepburn S, Heasman PA. The effect of smoking on periodontal treatment response: a review of clinical evidence. J Clin Periodontol. 2006;33(4):241-53. doi:10.1111/j.1600-051X.2006.00902.x

19. Patel RA, Wilson RF, Palmer RM. The effect of smoking on periodontal bone regeneration: a systematic review and meta-analysis. J Periodontol. 2012;83(2):143-55. doi:10.1902/jop.2011.110130

20. Fisher S, Kells L, Picard JP, Gelskey SC, Singer DL, Lix $\mathrm{L}$ et al. Progression of periodontal disease in a maintenance population of smokers and non-smokers: a 3-year longitudinal study. J Periodontol. 2008;79(3):461-8. doi:10.1902/jop.2008.070296

21. Matuliene G, Pjetursson BE, Salvi GE, Schmidlin K, Brägger $\mathrm{U}$, Zwahlen $\mathrm{M}$ et al. Influence of residual pockets on progression of periodontitis and tooth loss results after 11 years of maintenance. J Clin Periodontol. 2008;35(8):685-95. doi:10.1111/j.1600-051X.2008.01245.x

22. Ravald N, Johansson CS. Tooth loss in periodontally treated patients: a long-term study of periodontal disease and root caries. J Clin Periodontol. 2012;39(1):73-9. doi:10.1111/j.1600-051X.2011.01811.x

23. Pereira AL, Cortelli SC, Aquino DR, Franco GC, Cogo K, Rodrigues $\mathrm{E}$ et al. Reduction of salivary arginine catabolic activity through periodontal therapy. Quintessence Int. 2012;43(9):777-87.

24. Sardi JC, Duque C, Camargo GA, Hofling JF, Gonçalves RB. Periodontal conditions and prevalence of putative periodontopathogens and Candida spp. in insulin-dependent type 2 diabetic and non-diabetic patients with chronic periodontitis: a pilot study. Arch Oral Biol. 2011;56(10):1098-105. doi:10.1016/j.archoralbio.2011.03.017

25. Ashimoto A, Chen C, Bakker I, Slots J. Polymerase chain reaction detection of 8 putative periodontal pathogens in subgingival plaque of gingivitis and advanced periodontitis lesions. Oral Microbiol Immunol. 1996;11(4):266-73. doi:10.1111/j.1399-302X.1996.tb00180.x

26. Benkirane RM, Guillot E, Mouton C. Immunomagnetic PCR and DNA probe for detection and identification of Porphyromonas gingivalis. J Clin Microbiol. 1995;33(11):2908-912.

27. Slots J, Ashimoto A, Flynn MJ, Li G, Chen C. Detection of putative periodontal pathogens in subgingival specimens by $16 \mathrm{~S}$ ribosomal DNA amplification with the polymerase chain reaction. Clin Infect Dis. 1995;20(Suppl 2):S304-7. doi:10.1093/clinids/20.Supplement_2.S304

28. Donnelly SM, Sullivan DJ, Shanley DB, Coleman DC. Phylogenetic analysis and rapid identification of Candida dubliniensis based on analysis of ACT1 intron and exon sequences. Microbiology. 1999;145(8):1871-82. doi:10.1099/13500872-145-8-1871

29. Haffajee AD, Cugini MA, Dibart S, Smith C, Kent RL Jr, Socransky SS. The effect of SRP on the clinical and microbiological parameters of periodontal diseases. J Clin Periodontol. 1997;24(5):324-34. doi:10.1111/j.1600-051X.1997.tb00765.x

30. Lee J, Taneja V, Vassallo R. Cigarette smoking and inflammation: cellular and molecular mechanisms. J Dent Res. 2012;91(2):142-9. doi:10.1177/0022034511421200

31. Ryder MI, Fujitaki R, Lebus S, Mahboub M, Faia B, Muhaimin D et al. Alterations of neutrophil L-selectin and CD18 expression by tobacco smoke: implications for periodontal diseases. J Periodontal Res. 1998;33(6):359-68. doi:10.1111/j.1600-0765.1998.tb02211.x

32. Tymkiw KD, Thunell DH, Johnson GK, Joly S, Burnell $\mathrm{KK}$, Cavanaugh JE et al. Influence of smoking on gingival crevicular fluid cytokines in severe chronic periodontitis. J Clin Periodontol. 2011;38(3):219-28. doi:10.1111/j.1600-051X.2010.01684.x 
- Prevalence of periodontopathogens and Candida spp. in smokers after nonsurgical periodontal therapy - a pilot study

33. César-Neto JB, Duarte PM, Oliveira MC, Tambeli $\mathrm{CH}$, Sallum EA, Nociti FH Jr. Smoking modulates interleukin-6:interleukin-10 and RANKL:osteoprotegerin ratios in the periodontal tissues. J Periodontal Res. 2007;42(2):184-91. doi:10.1111/j.1600-0765.2006.00934.x

34. Guglielmetti MR, Rosa EF, Lourenção DS, Inoue G, Gomes EF, De Micheli G et al. Detection and quantification of periodontal pathogens in smokers and never-smokers with chronic periodontitis by real-time polymerase chain reaction. J Periodontol. 2014;85(10):1450-7. doi:10.1902/jop.2014.140048

35. Renvert S, Dahlén G, Wikström M. The clinical and microbiological effects of non-surgical periodontal therapy in smokers and nonsmokers. J Clin Periodontol. 1998;25(2):153-7. doi:10.1111/j.1600-051X.1998.tb02421.x

36. Williams DW, Walker R, Lewis MA, Allison RT, Potts AJ. Adherence of Candida albicans to oral epithelial cells differentiated by Papanicolaou staining. J Clin Pathol. 1999;52(7):529-31. doi:10.1136/jcp.52.7.529
37. Urzúa B, Hermosilla G, Gamonal J, Morales-Bozo I, Canals $\mathrm{M}$, Barahona $\mathrm{S}$ et al. Yeast diversity in the oral microbiota of subjects with periodontitis: Candida albicans and Candida dubliniensis colonize the periodontal pockets. Med Mycol. 2008;46(8):783-93. doi:10.1080/13693780802060899

38. Canabarro A, Valle C, Farias MR, Santos FB, Lazera M, Wanke B. Association of subgingival colonization of Candida albicans and other yeasts with severity of chronic periodontitis. J Periodontal Res. 2013;48(4):428-32. doi:10.1111/jre.12022

39. Machado AG, Komiyama EY, Santos SS, Jorge AO, Brighenti FL, Koga-Ito CY. In vitro adherence of Candida albicans isolated from patients with chronic periodontitis. J Appl Oral Sci. 2011;19(4):384-7. doi:10.1590/S1678-77572011005000014

40. Tamai R, Sugamata M, Kiyoura Y. Candida albicans enhances invasion of human gingival epithelial cells and gingival fibroblasts by Porphyromonas gingivalis. Microb Pathog. 2011;51:250-4. doi:10.1016/j.micpath.2011.06.009 